Marília da Glória Martins ${ }^{1}$

Graciete Helena Nascimento dos Santos

MÁrCia da SILVA SOUSA ${ }^{3}$

JanNe Eyre Fernandes BRTTO dA COSTA ${ }^{4}$

Vanda Maria FerRerRa SimÕes ${ }^{5}$

\title{
Associação de gravidez na adolescência e prematuridade
}

\author{
Association of pregnancy in adolescence and prematurity
}

Artigo Original

Palavras-chave

Gravidez na adolescência/complicações Trabalho de parto prematuro

Prematuro

Assistência perinatal

Keywords

Pregnancy in adolescence/complications Obstetric labor, premature Infant, premature Perinatal care

\section{Resumo}

OBJETIVO: Analisar a associação da gravidez na adolescência com prematuridade. MÉTODOS: Foram incluídas todas as pacientes que pariram num hospital terciário universitário do Maranhão, no período de julho a dezembro de 2006, alocando-as em dois grupos: adolescentes ( 10 a 19 anos de idade) e adultas (20 a 34 anos). As variáveis estudadas foram: escolaridade, situação conjugal, número de consultas no pré-natal, idade gestacional no início do pré-natal, duração da gestação, tipo de parto e peso ao nascer. Os dados foram processados no programa Epi-Info, versão 3.4.1, e foram analisadas as associações entre as variáveis pela razão dos produtos cruzados, a odds ratio (OR), com intervalo de confiança $(\mathrm{IC})$ de $95 \%$; utilizaram-se também modelos de regressão logística. $\bigcirc$ nível de significância adotado foi de 0,05. RESULTADOS: Foram avaliadas 1.978 pacientes. Verificou-se frequência de 25,4\% de partos em adolescentes, que apresentaram baixa escolaridade, ausência de companheiro, menor número de consultas no pré-natal, início tardio do pré-natal, baixo peso ao nascer (BPN) e prematuridade. Realizando a análise, tendo como variável desfecho a prematuridade, verificou-se nítida associação com baixo número de consultas do pré-natal (OR 3,0; IC95\% 2,2-4,0) e início tardio do pré-natal (OR 1,9; IC95\% 1,3-2,6), baixa escolaridade (OR 1,9; IC95\% 1,4-2,5) em relação com a adolescência (OR 1,5; IC95\% 1, 1-1,9). As adolescentes tiveram menor incidência de cesárea (33,3\%) que as adultas $(49,4 \%)$, com diferença significativa, além de menor associação com pré-eclâmpsia e desproporção cefalopélvica. CONCLUSÕES: A gravidez na adolescência esteve associada a início tardio do pré-natal e baixo número de consultas pré-natal, além de baixa escolaridade, BPN, prematuridade e menor incidência de desproporção cefalopélvica e pré-eclâmpsia.

\section{Abstract}

PURPOSE: To analyze the association of pregnancy in adolescence and prematurity. METHODS: The study included all the patients who delivered at a teaching hospital in Maranhão State, from July to December 2006. The patients were divided into two groups: adolescents ( 10 to 19 years old) and adults (20 to 34 years old). The variables studied were: educational level, marital status, number of prenatal visits, gestational age at the onset of prenatal care, duration of gestation, delivery route and birth weight. Statistical analysis was performed using the Epi-Info software, version 3.4. 1, and the associations between variables were analyzed by the odds ratio (OR), with a $95 \%$ confidence interval (Cl). Models of logistic regression were also used. The level of significance adopted was 0.05. RESULTS: The study evaluated 1,978 patients. The frequency of deliveries in adolescents was $25.4 \%$. This group presented low educational level, no mates, low number of prenatal visits, late onset of prenatal care, low birth weight and prematurity. In the analysis of prematurity as the outcome variable, there was a clear association with low number of prenatal visits (OR 3.0; $95 \% \mathrm{Cl} 2.2-4$.0) and late onset of prenatal care IOR 1.9; 95\% Cl 1.3-2.6) and low educational level (OR 1.9;95\% Cl 1.4-2.5) related to adolescence $(O R$ 1.5; $95 \% \mathrm{Cl}$ 1.1-1.9). The incidence of caesarean delivery was significantly lower among adolescents (33.3\%) than among adults $(49.4 \%)$, with a lower association with pre-eclampsia and cephalo-pelvic disproportion. CONCLUSIONS: Pregnancy in adolescence was associated with late onset of prenatal care and low number of visits, as well as low educational level, low birth weight, prematurity and a lower incidence of cephalo-pelvic disproportion and pre-eclampsia.
Correspondência:

Marilia da Glória Martins Av. 01 Qd E, Casa 21, Jardim Coetho Neto - Calhau CEP: $65066-240$

São Luis (MA), Brasil

Recebido

$22 / 12 / 2010$

Aceito com modificacōes $25 / 10 / 2011$
Serviço de Obstetrícia e Ginecologia do Hospital Universitário da Universidade Federal do Maranhão - UFMA - São Luís (MA), Brasil. ' Professora Associada da Disciplina de Obstetrícia do Departamento de Medicina III; Chefe do Serviço de Obstetrícia e Ginecologia do Hospital Universitário da Universidade Federal do Maranhão - UFMA - São Luís (MA), Brasil.

${ }^{2}$ Médica Obstetra do Serviço de Obstetrícia e Ginecologia do Hospital Universitário da Universidade Federal do Maranhão - UFMA São Luís (MA), Brasil.

${ }^{3}$ Médica Obstetra do Serviço de Obstetrícia e Ginecologia do Hospital Universitário da Universidade Federal do Maranhão - UFMA São Luís (MA), Brasil.

${ }^{4}$ Médica Obstetra do Serviço de Obstetrícia e Ginecologia do Hospital Universitário da Universidade Federal do Maranhão - UFMA São Luís (MA), Brasil.

5 Professora Adjunta da Disciplina de Semiologia do Departamento de Medicina I da Universidade Federal do Maranhão - UFMA São Luís (MA), Brasil.

Conflito de interesses: não há 
Introdução

Adolescência é o período compreendido entre os 10 e 19 anos, caracterizado por intenso crescimento e desenvolvimento, que se manifestam por marcantes transformações anatômicas, fisiológicas, mentais e sociais ${ }^{1,2} . \mathrm{Na}$ atualidade, gravidez no extremo inferior da vida reprodutiva tem sido objeto de preocupação, pois a gestação, o parto e a maternidade são situações que podem trazer múltiplas consequências emocionais, sociais e econômicas para a saúde da mãe adolescente e do seu filho. Com o aumento do número de gestantes adolescentes surgem grandes desafios na perinatologia, por conta dos resultados neonatais adversos encontrados em recém-nascidos de adolescentes ${ }^{3}$.

No mundo, aproximadamente $25 \%$ de mulheres têm seu primeiro filho antes de completados os 20 anos de idade, com taxas ainda mais altas em países em desenvolvimento. Segundo o censo de 2010, o Brasil registra 190.755.799 milhões de habitantes, sendo que 17,9\% estão no período da adolescência, assim, 17 milhões são mulheres adolescentes ${ }^{4}$. A população jovem (abaixo de 20 anos) constitui mais de um terço do total, a maior coorte de adolescentes de todos os tempos, respondendo por um milhão de gravidezes/ano. Assim, em nosso país, emerge o reconhecimento da gravidez na adolescência como um crescente problema de saúde pública.

Estudos realizados em diferentes regiões têm explorado o impacto da gravidez na adolescência sobre a mortalidade materna e neonatal. Um fato preocupante é que as complicações relacionadas à gravidez e ao parto estão entre as principais causas de morte de adolescentes no período de 15 a 19 anos de idade, em todas as cercanias do mundo 5 . Ao comparar as cifras com dados provenientes de mulheres adultas, observa-se que as adolescentes apresentam maior incidência de complicações médicas que envolvem tanto a mãe quanto o filho; dados recentes indicam que esses riscos são especialmente relevantes para as adolescentes mais jovens. As adolescentes têm $75 \%$ mais risco de ter parto prematuro que as mulheres adultas ${ }^{6,7}$.

Para a adolescente, a gravidez ocorre em um organismo que ainda está em desenvolvimento físico e emocional, e poderá apresentar problemas de crescimento e desenvolvimento, distúrbios emocionais e comportamentais, educacionais e de aprendizado, além de complicações na gravidez e problemas inerentes ao parto ${ }^{8}$. A jovem mãe geralmente não está habilitada para a nova função e, adicionalmente, encontra maiores dificuldades para continuar os estudos e inserir-se no mercado de trabalho ${ }^{6}$. A gravidez na adolescência é considerada um problema de saúde pública, uma vez que pode acarretar complicações obstétricas, com repercussões para a mãe e o recém-nascido, bem como problemas psicossociais e econômicos ${ }^{2,9}$.
As pesquisas apontam maior incidência de partos prétermos e de recém-nascidos de baixo peso nesse grupo de gestantes que, como é sabido, são importantes marcadores de morbidade e mortalidade neonatal e infantil, sendo a prematuridade responsável por cerca de $70 \%$ da taxa de mortalidade perinatal no Brasil ${ }^{3,6,7,10}$. O parto pré-termo é intercorrência obstétrica com maior frequência entre adolescentes em comparação com gestantes de outras faixas etárias ${ }^{11,12}$. O aumento dos índices de gravidez na adolescência pode contribuir para maior prevalência de parto pré-termo, apesar da etiologia dos aspectos obstétricos da prematuridade ser multifatorial. Dentro do universo das causas de parto pré-termo, as condições socioeconômicas adversas, a desnutrição, a anemia ferropriva, as infecções do trato geniturinário, primiparidade jovem e doença hipertensiva na gravidez são as intercorrências que interferem no equilíbrio materno-fetal, contribuindo para aumentar os percentuais de parto pré-termo e de recém-nascidos de baixo peso ${ }^{6}$.

A prematuridade constitui-se em um grande problema de saúde pública, por tratar-se de um determinante de morbimortalidade neonatal, principalmente em países em desenvolvimento. Crianças prematuras e com baixo peso ao nascer apresentam risco de mortalidade significativamente superior a crianças nascidas com peso maior ou igual a 2.500 g e duração da gestação maior ou igual a 37 semanas $^{11,13,14}$. Em países industrializados, a prematuridade é responsável por $70 \%$ da mortalidade neonatal e $75 \%$ da morbidade neonatal, além de contribuir com problemas de desenvolvimento do sistema nervoso, disfunção pulmonar e complicações visuais ${ }^{13,15}$.

Considerando o importante papel da prematuridade na mortalidade infantil no País, torna-se importante e necessário identificar as causas desse aumento por meio de estudos específicos. A partir da determinação dessas causas poderão ser planejadas intervenções que diminuam a ocorrência de partos prematuros e, consequentemente, as taxas de mortalidade infantil ${ }^{16}$.

\section{Métodos}

Foi feito um estudo observacional e analítico, realizado no Serviço de Obstetrícia e Ginecologia do Hospital Universitário da Universidade Federal do Maranhão (HU-UFMA), no período de julho a dezembro de 2006, nas enfermarias de puerpério, com a casuística total do período.

Foram entrevistadas todas as mulheres internadas nas enfermarias do puerpério no período de $1^{\circ}$ de julho a 31 de dezembro de 2006, perfazendo um total de 2.196 pacientes. As adolescentes (10-19 anos) foram comparadas com mulheres adultas (20-34 anos), assim foram excluídas 132 pacientes com idade $\geq 35$ anos. Foram incluídas as gestantes hígidas, que pariram nesse Serviço, com idade gestacional $\geq 22$ semanas, e conceptos com peso $\geq 500$ g e gestação única. 
Foram excluídas 42 pacientes com gestação múltipla e 44 com doenças preexistentes. A amostra final totalizou 1.975 pacientes.

Após a aplicação dos critérios de inclusão e exclusão, a leitura e o aceite, do termo de consentimento livre e esclarecido, aplicamos o questionário previamente confeccionado, com perguntas abertas e fechadas. A seguir, foram buscados os dados técnicos para complementar os itens do questionário nos prontuários e na Declaração de Nascidos Vivos. O estudo foi aprovado pelo Comitê de Ética em Pesquisa do HU-UFMA, respeitando todos os requisitos exigidos pela resolução 196/96 do Conselho Nacional de Saúde para estudos envolvendo seres humanos.

Efetuou-se análise univariada com distribuições de frequências e medidas de dispersão e tendência central. Utilizou-se o teste do $\chi^{2}$ para avaliar a significância das associações e, quando esse não foi aplicável, utilizou-se o teste exato de Fisher.

A idade gestacional foi definida com base na data da última menstruação e confirmada com o exame ultrassonográfico, de preferência de primeiro trimestre, quando disponível. A prematuridade foi definida como idade gestacional menor que 37 semanas no momento do parto ${ }^{17}$. Com relação à escolaridade, considerou-se baixa escolaridade (de zero a sete anos de estudo) e boa escolaridade (oito ou mais anos de estudo); com relação à situação conjugal, foram reunidas as pacientes com companheiro (casadas ou com união consensual) e aquelas sem companheiro (solteiras, divorciadas, viúvas); quanto ao atendimento pré-natal, considerou-se início tardio quando iniciado após o quarto mês de gestação comparando com início oportuno, quando iniciado antes disso. Quanto ao número de consultas, houve uma adaptação entre o que preconiza o Ministério da Saúde (pré-natal adequado com seis ou mais consultas) e o estudo realizado por Santos et al. ${ }^{10}$, que consideraram as seguintes categorias: pré-natal ausente, quando a gestante não realizou nenhuma consulta; pré-natal inadequado ou precário quando realizou de uma a três consultas; pré-natal regular ou intermediário quando realizou de quatro a seis consultas e pré-natal adequado quando a mulher compareceu a mais de seis consultas; quanto ao tipo de parto, normal (vaginal) ou operatório (cesárea); quanto ao peso do recém-nascido, peso adequado $(\geq 2.500 \mathrm{~g})$ ou baixo peso ao nascer $(<2.500 \mathrm{~g})$.

Para análises multivariadas, utilizaram-se modelos de regressão logística, tendo, como variável, resposta a prematuridade. Como variáveis independentes, foram incluídas as variáveis: faixa etária materna, escolaridade, número e consultas no pré-natal, idade gestacional no início do pré-natal e situação conjugal. Foram estimadas as odds ratio $(\mathrm{OR})$ brutas e respectivos intervalos de confiança (IC) de $95 \%$. O valor de $\mathrm{p}$ considerado estatisticamente significante foi $<0,05$. A análise dos dados foi realizada por meio do software estatístico de domínio público Epi-Info, versão 3.4.1, de 2007.

\section{Resultados}

Foram entrevistadas todas as mulheres atendidas no Serviço, no período de julho a dezembro de 2006. Após aplicados os critérios de exclusão, a amostra final totalizou 1.975 pacientes. Os resultados são apresentados em tabelas com números absolutos e relativos, e, com fins comparativos, as mulheres estão agrupadas por idade de 10 a 19 anos e de 20 a 34 anos, considerada faixa de idade ideal para procriação e utilizada como grupo controle. Com relação à escolaridade, os dados demonstram que a maioria dos partos ocorreu em mulheres com tempo de estudo de 8 a 11 anos, sendo $389(72,4 \%)$ adolescentes e $1.096(76,2 \%)$ adultas. As adolescentes com pouca escolaridade (menos de sete anos de estudo) representaram $26,1 \%$ do total de adolescentes, comparadas com, somente, $16,4 \%$ entre as adultas; o analfabetismo (igual a 0 anos de estudo), correspondeu a $0,3 \%$ do total das mulheres.

Quanto à situação conjugal, tanto nas adolescentes $(58,1 \%)$ quanto nas adultas $(58,5 \%)$, predominou a união consensual, onde não houve diferença percentual entre os dois grupos.

Com relação ao pré-natal, a maioria das pacientes iniciou o pré-natal até o quarto mês, as pacientes adultas em maior número, 1.264 (87,9\%), do que as adolescentes, onde $427(79,5 \%)$ o fizeram (Tabela 1$)$.

As informações relativas ao número de consultas no pré-natal indicam que a maioria das pacientes frequentou pré-natal regularmente ( 4 a 6 consultas), sendo 271

Tabela 1. Variáveis relativas ao atendimento pré-natal, parto e recém-nascido entre as mulheres atendidas no Serviço de Obstetrícia \& Ginecologia do Hospital Universitário da Universidade Federal do Maranhão, no período de $1^{0}$ de julho a 31 de dezembro de 2006

\begin{tabular}{|c|c|c|c|c|c|}
\hline \multirow{3}{*}{$\begin{array}{l}\text { Variáveis do pré-natal, } \\
\text { parto e recém-nascido }\end{array}$} & \multicolumn{4}{|c|}{ Idade materna } & \multirow{3}{*}{ Valor $p$} \\
\hline & \multicolumn{2}{|c|}{ 10-19 anos } & \multicolumn{2}{|c|}{ 20-34 anos } & \\
\hline & n & $\%$ & n & $\%$ & \\
\hline IG no início do PN* & & & & & $<0,0001$ \\
\hline 1 a 4 meses & 427 & 79,5 & 1.264 & 87,9 & \\
\hline 5 meses ou mais & 93 & 17,3 & 133 & 9,3 & \\
\hline Ignorado ou não fez & 17 & 3,2 & 41 & 2,8 & \\
\hline Número de consultas no PN & & & & & $<0,0001$ \\
\hline 0 & 11 & 2,0 & 21 & 1,5 & \\
\hline 1 a 3 & 104 & 19,4 & 164 & 11,4 & \\
\hline 4 a 6 & 271 & 50,5 & 743 & 51,7 & \\
\hline$\geq 6$ & 151 & 28,1 & 510 & 35,4 & \\
\hline Tipo de parto & & & & & $<0,0001$ \\
\hline Normal & 358 & 66,7 & 730 & 50,8 & \\
\hline Cesćreo & 179 & 33,3 & 708 & 49,2 & \\
\hline Idade Gestacional & & & & & 0,0006 \\
\hline $22-36$ semanas & 115 & 21,4 & 226 & 15,7 & \\
\hline $37-41$ semanas & 408 & 76,0 & 1.151 & 80,1 & \\
\hline$\geq 42$ semanas & 14 & 2,6 & 61 & 4,2 & \\
\hline Total & 537 & & 1.438 & & \\
\hline
\end{tabular}

IG: idade gestacional; PN: pré-natal. 
adolescentes $(50,5 \%)$ e 743 adultas $(51,7 \%)$ e, das que não tiveram nenhuma ou no máximo 3 consultas, $115(21,4 \%)$ eram adolescentes e 185 (12,9\%) adultas. Levando em consideração o critério definido pelo Ministério da Saúde, que considera adequado o pré-natal em que a paciente realiza no mínimo seis consultas, verificamos que $71,9 \%$ das adolescentes não realizaram um pré-natal adequado versus $64,5 \%$ das adultas. Entre as adolescentes, um número expressivo (110), perfazendo $20,5 \%$ das pacientes, não realizou o pré-natal ou o iniciou tardiamente (após o $4^{\circ}$ mês) e, entre as adultas, isso ocorreu somente com $174(12,1 \%)$ pacientes.

Considerando-se o tipo de parto, nota-se predominância discreta de parto normal $(55,1 \%)$, quando comparadas aos partos cesáreos $(44,9 \%)$. Em relação aos grupos etários, observa-se que, entre as adolescentes, os percentuais de cesáreas são menores, com $66,7 \%$ de parto normal versus $33,3 \%$ de cesáreas, sendo 66,2\% nas adolescentes de 15 a 19 anos e atingindo $80 \%$ de partos normais nas menores de 15 anos, quando comparadas às mulheres adultas, que ultimaram a gestação por parto operatório em maior proporção, $49,2 \%$ nas mulheres entre 20 e 34 anos, sendo $45,2 \%$ entre 20 e 24 anos, $48,8 \%$ na idade de 25 a 29 anos e chegando a 64,6\% na faixa entre 30 e 34 anos. É notável o valor crescente de partos cesáreos, por faixa etária, bem como o percentual decrescente de parto normal.

As variáveis significantes foram idade gestacional e peso ao nascer ( $\mathrm{p}=0,0006 \mathrm{e} \mathrm{p}=0,0062$, respectivamente) (Tabela 1). A prematuridade ocorreu em 17,3\% dos recémnascidos, o que significa dizer que, aproximadamente, de cada 30 mulheres, 5 delas não completaram as 37 semanas de gestação, sendo o maior percentual entre as adolescentes, com 21,4 versus $15,7 \%$ entre as adultas.

Com relação ao peso do recém-nascido, $15,8 \%$ de todas as mulheres tiveram filhos com peso $<2500 \mathrm{~g}$, sendo $19,9 \%$ entre as adolescentes e $14,3 \%$ entre as adultas; $79,8 \%$ dos recém-nascidos apresentaram peso adequado, com resultados semelhantes entre os grupos $(78,4$ versus $80,4 \%)$ e $4,4 \%$ dos conceptos nasceram com mais de $4.000 \mathrm{~g}$ (1,6\% de adolescentes versus 5,3\% de adultas).

Todas as variáveis estudadas mostraram-se estatisticamente significantes, sendo o início tardio do pré-natal (OR 1,9), pré-natal inadequado, com poucas consultas (OR 1,8), e ausência de companheiro (OR 1,9), as mais relevantes. Quanto ao parto cesáreo, a adolescência se mostrou como fator de proteção (OR 0,5) (Tabela 2).

Com relação ao desfecho prematuridade, verificou-se associação com o baixo número de consultas pré-natal, menos de quatro consultas, quando a gestante tem três vezes mais chance de ter concepto prematuro (OR 3,2; IC95\% 2,4-4,2), bem como com baixa escolaridade da mãe (OR 1,6; IC95\% 1,2-2,1), além da associação com a adolescência per se (OR 1,4) (Tabela 3).
Tabela 2. Comparação das razões de chances $(O R=$ odds ratio) entre adolescentes $(10$ a 19 anos) e adultas (20 a 34 anos) para algumas variáveis

\begin{tabular}{lc|c|c|c|c}
\hline \multicolumn{7}{c}{ Adolescentes $\times$ Adultas } & & & \\
\hline & $10-19$ & $20-34$ & OR & IC95\% & Valor p \\
\hline Ausência de & 182 & 305 & 1,9 & $1,5-2,3$ & $<0,0001$ \\
companheiro & 355 & 1133 & & & \\
Baixa escolaridade & 140 & 236 & 1,8 & $1,4-2,2$ & $<0,0001$ \\
\hline \multirow{4}{*}{ Parło cesáreo } & 397 & 1202 & & & \\
& 179 & 708 & 0,5 & $0,4-0,6$ & $<0,0001$ \\
\hline Prematuridade & 358 & 730 & & & \\
& 115 & 226 & 1,4 & $1,1-1,8$ & 0,0029 \\
\hline \multirow{4}{*}{ Peso<2500 g } & 422 & 1212 & & & \\
\hline Pré-natal inadequado & 107 & 205 & 1,5 & $1,1-1,9$ & 0,0021 \\
ou ausente & 430 & 1233 & & & \\
Pré-natal de início tardio & 386 & 928 & 1,4 & $1,1-1,7$ & $<0,0021$ \\
\hline
\end{tabular}

IC: intervalo de confiança

Tabela 3. Análise multivariada dos fatores associados à ocorrência de prematuridade na amostra estudada

\begin{tabular}{|c|c|c|c|c|}
\hline Variável & Categoria & OR & Significância & IC95\% \\
\hline Faixa etária & $\begin{array}{l}\text { Adolescentes } \\
\text { Adultas }\end{array}$ & $\begin{array}{l}1,4 \\
1,0\end{array}$ & 0,003 & $1,1-1,8$ \\
\hline Início do pré-natal & $\begin{array}{c}\text { Nenhum ou } \geq 5 \text { meses } \\
\text { Até } 4 \text { meses }\end{array}$ & $\begin{array}{l}1,3 \\
1,0\end{array}$ & 0,03 & $1,0-1,9$ \\
\hline Consultas de pré-natal & $\begin{array}{c}0 \text { a } 3 \\
4 \text { ou mais }\end{array}$ & $\begin{array}{l}3,1 \\
1,0\end{array}$ & $<0,05$ & $2,3-4,2$ \\
\hline Escolaridade & $\begin{array}{l}0 \text { a } 7 \text { anos } \\
8 \text { ou mais }\end{array}$ & $\begin{array}{l}1,6 \\
1,0\end{array}$ & $<0,05$ & $1,2-2,1$ \\
\hline Situação conjugal & $\begin{array}{l}\text { Sem companheiro } \\
\text { Com companheiro }\end{array}$ & $\begin{array}{l}1,0 \\
1,0\end{array}$ & 0,9 & $0,7-1,3$ \\
\hline
\end{tabular}

OR: odds ratio; IC: intervalo de confiança

\section{Discussão}

A prevalência de partos em adolescentes verificada nesta pesquisa foi de $25,4 \%$, número superior à média nacional (22\%) e aos encontrados em Campinas (SP) $(17,8 \%)^{15}$, e em Montes Claros (MG) $(21,5 \%)^{7}$, estudos baseados em dados do Sistema de Informações de Nascidos Vivos (SINASC); porém, um pouco abaixo do resultado encontrado por Simões et al. ${ }^{18}(29,4 \%)$, em pesquisa realizada na mesma cidade do atual estudo, e por Amorim et al. ${ }^{19}$ (27,2\%), em maternidade-escola da Paraíba $(27,2 \%)$, região com características socioeconômicas parecidas, onde predominam o baixo nível de escolaridade das adolescentes, dos pais e a dificuldade de acesso aos serviços de saúde, fatores de risco para o crescimento do número de adolescentes grávidas ${ }^{18-20}$.

Essas taxas são consideradas muito altas quando comparadas com as de países desenvolvidos, como o Canadá, França e Suécia, que apresentam respectivamente, 11,6\% e $4 \%{ }^{21}$. Nos Estados Unidos, em 2001, a proporção de gravidez em mulheres de 10 a 19 anos foi de $4,1 \%$, apresentando redução de $24 \%$ desde $1990^{22}$. 
A frequência de gravidez na adolescência tem grande variação e depende da situação social e econômica da região, sendo mais incidente nas regiões menos desenvolvidas e menos urbanizadas ${ }^{12,23}$. De acordo com os dados do Registro Civil 2005, 19,9\% das crianças que nasceram naquele ano foram de mães adolescentes. Não obstante, esse aumento relativo pode estar também influenciado pelo decréscimo acentuado da fecundidade nas faixas etárias a partir de 25 anos. Essa proporção se mostrou mais elevada na Região Norte, com destaque para Tocantins, Pará e Acre, além do Maranhão na região Nordeste. No outro extremo, o Distrito Federal, São Paulo, Rio de Janeiro e Rio Grande do Sul, apresentam valores abaixo da média nacional. Entre 1995 e 2005, o Piauí e o Ceará foram os estados que tiveram os maiores crescimentos relativos da proporção de nascidos vivos de mães adolescentes, com $54,1 \%$ e $39,7 \%$, respectivamente ${ }^{4}$.

Com relação à escolaridade, $75,1 \%$ do total de mulheres pesquisadas verificou-se que elas tinham de 8 a 11 anos de estudo. Já as adolescentes com pouca escolaridade, até 7 anos de estudo, representaram 26,0\% do total de adolescentes versus $16,4 \%$ entre as adultas, uma incidência de baixa escolaridade menor que a encontrada em estudo realizado em Maceió, que encontrou elevada taxa de baixa escolaridade, analfabetas ou com menos de quatro anos de estudo entre as mulheres participantes da pesquisa $(90,8 \%$ entre adolescentes e $71,6 \%$ das adultas) ${ }^{23}$. Estudo de Campinas mostrou que $48,4 \%$ das adolescentes tinham escolaridade de até sete anos de estudo em relação a $43,3 \%$ das adultas ${ }^{9}$. A baixa escolaridade pode ser devido ao abandono escolar, que ocorre frequentemente em decorrência da gravidez, pois para aquelas que frequentam a escola, a gravidez leva, na maioria das vezes, a adolescente a abandonar os estudos temporária ou definitivamente.

Segundo dados da United Nations Children's Fund (UNICEF) $^{5}$, em 2009, das meninas que abandonaram a escola, 28\% fizeram pelo fato de estarem grávidas. Em estudo realizado em São José do Rio Preto, foi constatado que, para $75 \%$ das adolescentes, a gravidez foi motivo para deixarem os estudos ${ }^{24}$. É frequente a relação entre gravidez e abandono escolar ${ }^{5,19,24}$, levando a um agravamento das condições socioeconômicas dessas adolescentes, limitando suas possibilidades na qualificação e inserção no mercado de trabalho, cada vez mais exigente, gerando uma dependência do companheiro ou da família ${ }^{14,24}$.

Educação e urbanização são importantes determinantes de baixa incidência de gravidez em adolescentes ${ }^{19}$. A educação das adolescentes pode fortalecê-las a decidir sobre suas próprias vidas e desenvolver um comportamento planejado ${ }^{9,13,22}$. Quanto menor a escolaridade, maior a dificuldade de entendimento da necessidade de cuidados especiais no decorrer da gravidez, levando ao inicio tardio e ausência de pré-natal, alimentação inadequada e hábitos e vícios incompatíveis com a gravidez ${ }^{25}$.

Com relação à situação conjugal, houve predominância de mulheres que se declararam com união consensual (em torno de $58 \%$ nos dois grupos), $66,1 \%$ entre as adolescentes e $78,8 \%$ entre as adultas, além de maior percentual de solteiras entre as adolescentes $(33,9 \%)$, do que entre as adultas $(21,2 \%)$. Em estudo populacional americano verificou-se frequência de 20,6\% de adolescentes e 54,5\% de adultas que se declararam casadas ${ }^{26}$.

Verificou-se maior incidência de parto pré-termo entre as adolescentes, $(21,4 \%)$ em relação às adultas $(15,7 \%)$, inclusive na faixa de prematuridade extrema (22 a 33 semanas), quando ainda há imaturidade pulmonar (10,6\% entre as adolescentes e 5,6\% entre as mulheres entre 20 e 34 anos). A incidência de prematuridade na adolescência foi maior que o observado por Carniel et al. ${ }^{9}$, em estudo realizado em Campinas (7,5\%), porém menor do que a encontrada por Goldenberg et al. ${ }^{7}$, em Montes Claros (MG) e Chalem na periferia de São Paulo, que verificou percentual de $32,9 \%$ e $27 \%$, respectivamente, entre adolescentes de 10 a $19 \operatorname{anos}^{7,9,22}$. Esse percentual elevado de prematuridade entre adolescentes vem se mantendo ao longo dos anos em nosso meio, visto que, Simões et al. ${ }^{18}$ encontraram $22,9 \%$ de partos prematuros entre pacientes de 13 a 19 anos e 11,2\% entre aquelas de 20 a 24 anos.

Apesar da magnitude do problema, não está nítido se esses resultados dependem de fatores biológicos ou são unicamente a consequência de fatores sociodemográficos que se associam com a gravidez na adolescência. A imaturidade biológica poderia ser a explicação desses resultados adversos; essa imaturidade tem dois aspectos: por um lado a idade ginecológica jovem (definida como a concepção nos dois primeiros anos após a menarca) e por outro o risco de engravidar antes de haver completado o seu desenvolvimento. A imaturidade do suprimento sanguíneo do útero ou do colo uterino pode predispor as mães muito jovens a infecções subclínicas, aumento na produção de prostaglandinas com consequente aumento na incidência de partos prematuros. De outro modo, as mães adolescentes que continuam crescendo poderiam competir com o feto por nutrientes em detrimento do mesmo ${ }^{25}$.

Nesta pesquisa, realizada a análise multivariada tendo como desfecho a prematuridade, foi significante a associação com baixa frequência às consultas de pré-natal (OR 3,1; IC95\% 2,3-4,2) e baixa escolaridade (OR 1,6; IC95\% 1,2-2,1), além da adolescência per se (OR 1,4), contrariando estudo realizado em São Paulo onde a assistência pré-natal adequada pareceu diminuir a frequência de baixo peso ao nascer em filhos de mães adolescentes, porém não interferiu na frequência da prematuridade ${ }^{14}$. 
No que diz respeito ao atendimento pré-natal, nesta pesquisa verificamos também que $87,9 \%$ das pacientes adultas, entre 20 e 34 anos, iniciaram o pré-natal no primeiro trimestre da gestação, enquanto entre as adolescentes esse percentual foi de $79,5 \%$, resultado bom em relação a outros estudos, como o de Gama et al. ${ }^{27}$ que verificaram entre as puérperas de baixa renda um percentual de $10,3 \%$ de pacientes adolescentes sem consultas ou com início após o sexto mês, aproximadamente a metade do que foi encontrado em estudo analisando pacientes sem consulta ou início do quinto mês em diante $(20,4 \%)^{16}$. Estudo realizado em Maceió também encontrou uma incidência alta de $54,8 \%$ de adolescentes que realizaram menos de seis consultas no pré-natal ${ }^{28}$. No trabalho de Goldenberg et al. ${ }^{7}$, verificaram, que somente $28,5 \%$ das adolescentes realizaram pré-natal adequado. Os achados deste estudo são concordantes com estudo realizado no município de Campinas (SP), que relaciona gestação na adolescência com a baixa adesão ao pré-natal ${ }^{9}$.

Suzuki et al. ${ }^{14}$, em estudo em hospitais públicos de São Paulo, constatou que a gravidez na adolescência esteve relacionada com nascimentos de crianças prematuras (31,4\% no grupo de mães adolescentes e $8,6 \%$ no grupo de mães adultas, $\mathrm{p}=0,01)$. Em estudo em maternidade de Vitória (ES) verificou-se menores índices, 5,8\%, talvez devido à adesão pré-natal satisfatória, com seis ou mais consultas que chegaram a $80,7 \%$ nas adolescentes tardias ${ }^{3}$. $\mathrm{Na}$ maioria dos estudos, os autores referem-se a baixa adesão ao pré-natal, chegando inclusive a percentuais na ordem de $23 \%$. As diferenças geográficas, o nível de educação materna e as particularidades de cada população podem justificar os resultados dos percentuais diferentes encontrados na literatura ${ }^{3,10,19}$.

Em estudo realizado em Campinas (SP), com dados do SINASC, verificou-se que mais de um terço das adolescentes $(35,2 \%)$ compareceu em menos de sete consultas de pré-natal, enquanto $64,8 \%$ fizeram sete ou mais consultas, ao contrário da maioria das adultas $(76,5 \%)$ que fizeram o número adequado de consultas preconizado pelo Programa de Assistência Integral à Saúde da Mulher (PAISM) .

Gestantes adolescentes frequentam menos as consultas no período pré-natal ou ingressam tardiamente nesse acompanhamento. Vários fatores podem ser responsabilizados, desde a dificuldade de assumir a gestação, conflitos familiares, bem como o desconhecimento da importância dessa assistência. Uma questão que merece destaque é a estreita relação dos efeitos adversos à gestação com a assistência prestada no pré-natal. $\mathrm{O}$ acompanhamento médico adequado durante a gestação pode ser visto como uma política compensatória de saúde, cabendo a ele o papel de minimizar o efeito das desigualdades socioeconômicas ${ }^{26}$.
Os resultados mostraram que o número de consultas pré-natais exerce forte influência na ocorrência ou não de nascimentos prematuros. No presente estudo, a avaliação sobre a adequação do pré-natal restringiu-se ao número de consultas realizadas, considerando-se sete consultas ou mais como adequadas. Estudos verificaram que, com o aumento do número de consultas pré-natais, de nenhuma a três para sete ou mais, há redução significativa da prevalência de baixo peso e/ou pré-termo (de $14 \%$ para $4 \%)^{11,14}$. Esses resultados corroboram o presente estudo.

A atenção pré-natal é reconhecidamente importante na investigação de riscos biológicos de gestantes adolescentes, tais como colo curto, infecções, nutrição inadequada e abuso de drogas ilícitas, fatores implicados na ocorrência de parto prematuro. O pré-natal poderia também ser importante no sentido de oferecer suporte psicossocial nas dificuldades e situações de estresse, nas quais as mães adolescentes se encontram. Sofrimento emocional é reconhecido como causador de distúrbios endócrinos e parto pré-termo, e reduzindo esse estresse poderíamos conduzir a gravidez para resultados mais favoráveis. Suporte psicossocial de mães adolescentes pode prevenir marginalização econômica, educacional e social e não se limitar apenas ao atendimento médico necessário. Além do mais, como parturientes adolescentes são mais, frequentemente, desempregadas que outras mulheres de sua idade, suas crianças podem necessitar de suporte e vigilância adicional ${ }^{28}$.

Embora, teoricamente, a imaturidade biológica pudesse predispor o maior número de distocia e partos laboriosos, encontramos menor incidência de partos operatórios entre as adolescentes $(33,3 \%$ versus $49,2 \%$ entre as adultas), em concordância com os resultados encontrados em Montes Claros, que observaram 20\% de incidência de cesárea entre as adolescentes e $35 \%$ em idade adulta ${ }^{11}$, e similar ao observado em Maceió, onde se verificou 31,2\% de cesárea em pacientes abaixo de 20 anos e 42,8\% entre aquelas de 20 a 30 anos $^{13}$.

Em estudo realizado em Campinas, os partos vaginais $(63,8 \%)$ apresentaram maior percentual que os partos cesáreos para as menores de 20 anos, embora sua frequência tenha sido maior do que nas mulheres adultas $(45 \%)^{13}$. Ficou evidenciado no presente estudo que o grupo de adolescentes é protegido dos partos operatórios, achado semelhante a outros estudos. Uma provável explicação seria a alta taxa de partos prematuros e baixo peso ao nascer em adolescentes que poderiam estar associados com maior chance de parto via vaginal e a menor incidência de cesárea, bem como a política de incentivo do Sistema Único de Saúde em diminuir os partos operatórios, a fim de minimizar e otimizar a recuperação das mães.

Conclui-se, nesta pesquisa, que entre os principais fatores relacionados à prematuridade estão a 
adolescência e a baixa adesão às consultas de pré-natal. A complexa natureza de cuidados intensivos de crianças prematuras demanda profissionais de saúde altamente qualificados e altos custos de tratamento. Isso nos faz raciocinar que a adoção de políticas que visem o esclarecimento da importância das consultas pré-natal iniciadas precocemente, com equipe multiprofissional, profissionais qualificados para o atendimento de gestantes adolescentes é enfoque primordial na prevenção da gravidez na adolescência, uma vez que a gravidez nesse período da vida tem sérias implicações biológicas, familiares, emocionais e econômicas.

\section{Referências}

1. Chelala CA. La salud de los adolescentes y los jóvenes em las Américas: escribiendo el futuro. Washington (DC): Organización Panamericana de la Salud; 1995. (OPS Comunicación para la Salud, 6).

2. Yallez MEHD, Franco RC, Michelazzo D. Gravidez na adolescência: uma proposta para prevenção. Rev Bras Ginecol Obstet. 2009;31(10):477-9.

3. Rocha RCL, Souza E, Guazzeli CAF, Chambô Filho A, Soares EP, Nogueira E. Prematuridade e baixo peso entre recém-nascidos de adolescentes primíparas. Rev Bras Ginecol Obstet. 2006;28(9):530-5.

4. Instituto Brasileiro de Geografia e Estatística (IBGE). Síntese de indicadores sociais. Rio de Janeiro: IBGE; 2010. (Estudos e pesquisas: Informação Demográfica e Socioeconômica, 19).

5. UNICEF [Internet]. Situação mundial da infância 2011 . Adolescência: uma fase de oportunidades. Caderno Brasil. 2011 [citado 2011 Jun]. Disponível em: <http://www.unicef.org/brazil/pt/br_cadernoBR_ SOWCR 11 (3).pdf>.

6. Conde-Agudelo A, Belizán JM, Lammers C. Maternal-perinatal morbidity and mortality associated with adolescent pregnancy in Latin America: cross-sectional study. Am J Obstet Gynecol. 2005; 192(2):342-9.

7. Goldenberg P, Figueiredo MCT, Silva RS. Gravidez na adolescência, pré-natal e resultados perinatais em Montes Claros, Minas Gerais, Brasil. Cad Saúde Pública. 2005;21 (4):1077-86.

8. GalloJHS. Gravidez na adolescência: a idade materna, consequências e repercussões. Rev Bioética. $2011 ; 19(1): 179-95$.

9. Carniel EF, Zanolli ML, Almeida CAA, Morcillo AM. Características das mães adolescentes e de seus recém-nascidos e fatores de risco para a gravidez na adolescência em Campinas, SP, Brasil. Rev Bras Saúde Matern Infant. 2006;6(4):419-26.

10. Santos GHN, Martins MG, Sousa MS. Gravidez na adolescência e fatores associados com baixo peso ao nascer. Rev Bras Ginecol Obstet. 2008;30(5):224-31

11. Cascaes AM, Gauche H, Baramarchi FM, Borges CM, Peres KG. Prematuridade e fatores associados no Estado de Santa Catarina, Brasil, no ano de 2005: análise dos dados do Sistema de Informações sobre Nascidos Vivos. Cad Saúde Pública. 2008;24(5):1024-32.

12. Duarte $C M$, Nascimento VB, Akerman M. Gravidez na adolescência e exclusão social: análise de disparidades intra-urbanas. Rev Panam Salud Pública. 2006; 19(4):236-43.

13. Escobar GJ, Clark RH, Greene JD. Short-term outcomes on infants born at 35 and 36 weeks gestation: we need to ask more questions. Semin Perinatol. 2006;30(1):28-33.

14. Suzuki CM, Ceccon MEJ, Falcão MC, Vaz FAC. Análise comparativa da frequência de prematuridade e baixo peso entre filhos de mães adolescentes e adultas. Rev Bras Crescimento Desenvolv Hum. 2007;17(3):95-103.

15. Ananth CV, Joseph KS, Demissie K, Vintzileos AM. Trends in twin preterm birth subtypes in the United States, 1989 through 2000: impact on perinatal mortality. Am J Obstet Gynecol. 2005; 193/3 Pt 2):1076-82.

16. Silveira MF, Santos IS, Barros AJD, Matijasevich A, Barros FC, Victora CG. Aumento da prematuridade no Brasil: revisão de estudos de base populacional. Rev Saúde Pública. 2008;42(5):957-64.

17. WHO: recommended definitions, terminology and format for statistical tables related to the perinatal period and use of a new certificate for cause perinatal deaths. Modifications recommended by FIGO as amended October 14, 1976. Acta Obstet Gynecol Scand. 1977; 56(3):247-53.

18. Simões VMF, Silva AAM, Bettiol H, Lamy-Filho F, Tonial SR, Mochel EG. Características da gravidez na adolescência em São Luis, Maranhão. Rev Saúde Pública. 2003;37(5):559-65.

19. Amorim MMR, Lima LA, Lopes CV, Araújo DKL, Silva JGG, César $L C$, et al. Fatores de risco para a gravidez na adolescência em uma maternidade-escola da Paraíba: estudo caso-controle. Rev Bras Ginecol Obstet. 2009;31(8):404-10.

20. Brasil. Ministério da Saúde. Secretaria de Gestão Estratégica e Participativa. Gravidez na adolescência. Painel Indicadores SUS. 2006; 1(1): 19

21. Amaya J, Borrero C, Ucrós S. Estudio analítico del resultado del embarazo en adolescentes y mujeres de 20 a 29 años en Bogotá. Rev Colomb Obstet Ginecol. 2005;56(3):216-24.

22. Chalem E, Mitsuhiro SS, Ferri CP, Barros MCM, Guinsburg R, Laranjeira R. Gravidez na adolescência: perfil sócio-demográfico e comportamental de uma população da periferia de São Paulo, Brasil. Cad Saúde Pública. 2007;23(1):177-86.

23. Kassar SB, Lima MC, Albuquerque MFM, Barbieri MA, Gurgel $R Q$. Comparações das condições socioeconômicas e reprodutivas entre mães adolescentes e adultas jovens em três maternidades públicas de Maceió, Brasil. Rev Bras Saúde Matern Infant. 2006:6(4):397-403.

24. Faria DGS, Zanetta DMT. Perfil de mães adolescentes de São José do Rio Preto/Brasil e cuidados na assistência pré-natal. Arq Ciênc Saúde. 2008;15(1):17-23.

25. Ramos HAC, Cuman RKN. Fatores de risco para prematuridade: pesquisa documental. Esc Anna Nery Rev Enferm. 2009;13(2):297304

26. Chen XK, Wen SW, Fleming N, Demissie K, Rhoads GG, Walker $M$. Teenage pregnancy and adverse birth outcomes: a large population based retrospective cohorte study. Int J Epidemiol. 2007;36(2):368-73.

27. Gama SGN, Szwarcwald CL, Leal MC. Experiência de gravidez na adolescência, fatores associados e resultados perinatais entre puérperas de baixa renda. Cad Saúde Pública. 2002;18(1):153-61.

28. Raatikainen K, Heiskanen N, Verkasalo PK, Heinonen S. Good outcome of teenage pregnancies in high-quality maternity care. Eur J Public Health. 2005; 16(2):157-61. 